\title{
Three-Dimensional Trapping of Individual Rydberg Atoms in Ponderomotive Bottle Beam Traps
}

\author{
D. Barredo®, V. Lienhard, P. Scholl, S. de Léséleuc $\odot$, T. Boulier, A. Browaeys, and T. Lahaye $\odot$ \\ Laboratoire Charles Fabry, Institut d'Optique Graduate School, CNRS, Université Paris-Saclay, \\ 91127 Palaiseau Cedex, France
}

(Received 10 September 2019; published 16 January 2020)

\begin{abstract}
We demonstrate three-dimensional trapping of individual Rydberg atoms in holographic optical bottle beam traps. Starting with cold, ground-state ${ }^{87} \mathrm{Rb}$ atoms held in standard optical tweezers, we excite them to $n S_{1 / 2}, n P_{1 / 2}$, or $n D_{3 / 2}$ Rydberg states and transfer them to a hollow trap at $850 \mathrm{~nm}$. For principal quantum numbers $60 \leq n \leq 90$, the measured trapping time coincides with the Rydberg state lifetime in a $300 \mathrm{~K}$ environment. We show that these traps are compatible with quantum information and simulation tasks by performing single qubit microwave Rabi flopping, as well as by measuring the interaction-induced, coherent spin-exchange dynamics between two trapped Rydberg atoms separated by $40 \mu \mathrm{m}$. These results will find applications in the realization of high-fidelity quantum simulations and quantum logic operations with Rydberg atoms.
\end{abstract}

DOI: 10.1103/PhysRevLett.124.023201

Neutral atoms excited to Rydberg states are an attractive platform for scalable quantum simulation and computation $[1,2]$. The strong, controllable interactions between these states can be used to implement high-fidelity quantum gates, or to engineer various types of spin Hamiltonians difficult to study on classical computers [3]. These ideas have been intensively explored in the last years and several milestones have been achieved [4]. Prominent examples of this progress are the demonstration of strong optical nonlinearities [5], single-photon sources [6], conditional phase shifters [7], single-photon transistors [8,9], the experimental realizations of two-qubit gates [10-14], or the quantum simulations of spin models with tens of particles in optical lattices [15-17] and in arrays of optical tweezers [18-21].

In none of the above experiments were the Rydberg atoms trapped. However, controlling the motion of Rydberg atoms during gate operation and in quantum simulations is advantageous, since finite atom temperatures and mechanical forces due to the interactions between the particles ultimately limit quantum state fidelities [4,22] and the available time for coherent dynamics [19,23,24]. Rydberg trapping is also a prerequisite for precision measurements of fundamental constants using circular Rydberg states [25,26] or positronium [27].

To date, clouds of Rydberg atoms have been confined to three-dimensional, millimeter-size regions using static magnetic [28-30] or electric fields [31,32]. In an inhomogeneous ac electric field that oscillates faster than any internal frequency of the Rydberg atom, the weakly bound Rydberg electron experiences an oscillating force that can be used for trapping of the Rydberg atom [33]. The socalled ponderomotive potential, which is the time-averaged kinetic energy of the nearly free Rydberg electron oscillating in the laser field, is proportional to the light intensity. Therefore, to obtain a 3D trap, one must create a dark region surrounded by light in all directions; since the atom trapping arises mainly from the ponderomotive potential experienced by the electron, such traps can be used to confine Rydberg states whatever their $n, l, j, m_{j}$ quantum numbers.

Rydberg atoms have been confined in the tight potentials of ponderomotive optical lattices [33,34], but so far only in one dimension. Here we go beyond those initial demonstrations to show three-dimensional trapping of cold individual Rydberg atoms in micron-size optical potentials $[35,36]$. We use holography to create bottle beam (BOB) traps $[37,38]$ which are deterministically loaded with single Rydberg atoms. We characterize the depth and trapping frequencies of these traps and observe that the trapping time, for principal quantum numbers in the range $60 \leq n \leq 90$, is mainly limited by the Rydberg states lifetime in the presence of blackbody radiation at $300 \mathrm{~K}$. Finally, we illustrate the compatibility of these traps with quantum simulation by driving Rabi flopping between different Rydberg states, and by observing the coherent exchange of internal states induced by the dipole-dipole interaction for two atoms confined in BOB traps separated by $40 \mu \mathrm{m}$.

The setup is based on the one described in Ref. [39]. Briefly, we use a spatial light modulator (SLM) to create arbitrary arrays of micron-size optical tweezers (Fig. 1). To generate the BOB traps that will host Rydberg atoms, we follow the procedure of Refs. [38,40]. We use another SLM to imprint on another light beam at $850 \mathrm{~nm}$ a phase pattern, composed of two terms added modulo $2 \pi$ : (i) the standard 
hologram to create the point traps, and (ii) a centered disk of radius $r_{0}$, where the phase is shifted by $\pi$. The value of $r_{0}$ is adjusted such that, for each trap, destructive interference occurs between the central and outer parts of the Gaussian beam at the focal point, thus creating the needed dark region surrounded by light in all directions [41]. The local maximum of intensity encountered when moving away from the origin is smallest ( $\sim 10 \%$ of the maximal intensity) on two "escape cones" originating from the trap center. By changing the diameter of the beam impinging on the SLM and adjusting $r_{0}$ accordingly, the size of the trapping region can be tuned. An example of a BOB trap created this way is shown in Fig. 1 (inset), where we used an electrically tunable lens in the imaging path to record the 3D light intensity distribution near the focal plane of the aspherical lens. To allow for efficient transfer of the atoms between the two different traps (for ground-state and Rydberg atoms), the light from the BOB SLM is then overlapped with the standard optical tweezers beam using a polarizing beam splitter (PBS), ensuring that both traps are at the same position.

Far from resonances and in a Born-Oppenheimer-like approximation, the Rydberg atom trapping potential in a light field of intensity $I$ and angular frequency $\omega_{L}$ can be described as [42]

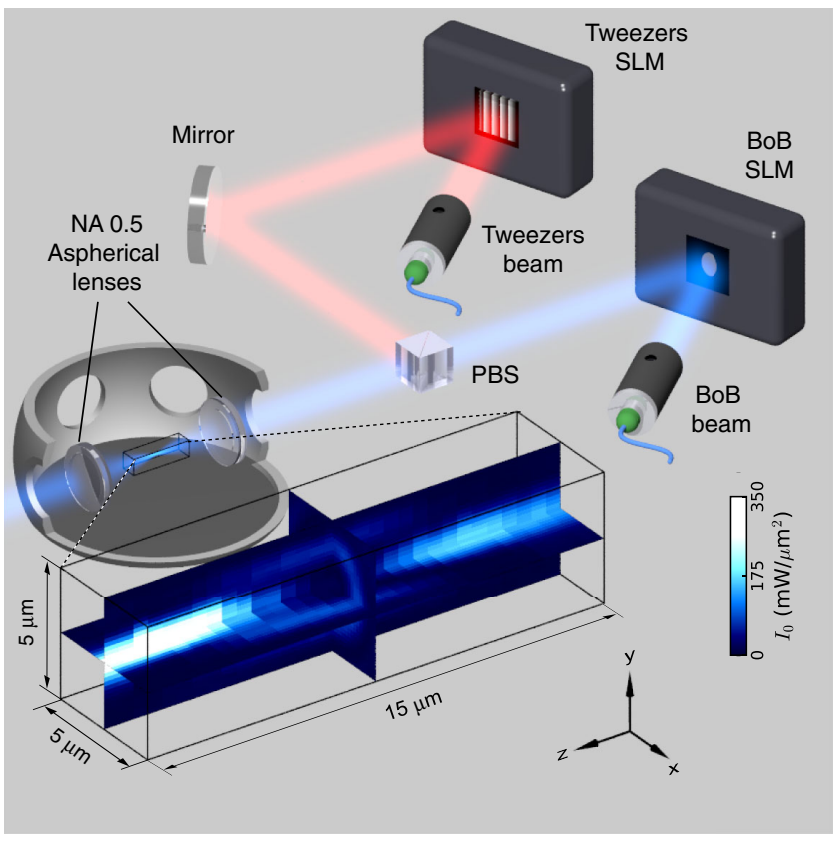

FIG. 1. Experimental setup. We use a spatial light modulator (SLM) and a high NA aspherical lens to generate a BOB trap for Rydberg atoms (blue beam, see text). The enlargement shows cuts of the reconstructed light intensity distribution near the focal plane, measured using an electrically tunable lens (not shown) to scan the images along the optical axis. To load the BOB trap, we use regular optical tweezers (red beam) created by light reflected from a second SLM and superimposed onto the BOB trap beam using a polarizing beam splitter.

$$
U_{n l j m_{j}}(\boldsymbol{R})=\int d^{3} r V_{P}(\boldsymbol{R}+r)\left|\psi_{n l j m_{j}}(\boldsymbol{r})\right|^{2} .
$$

Here, $V_{P}(\boldsymbol{r})=e^{2} I(\boldsymbol{r}) /\left(2 m_{e} \epsilon_{0} c \omega_{L}^{2}\right)$ is the repulsive ponderomotive shift experienced by the nearly free electron of mass $m_{e}$ and charge $e, \psi$ is the Rydberg wave function, $\boldsymbol{R}$ is the center of mass coordinate of the atom, and $\boldsymbol{r}$ is the relative position of the Rydberg electron.

An example of such calculation for the $84 S_{1 / 2}$ Rydberg state is shown in the cut displayed in Fig. 2(b), calculated from the measured intensity displayed on Fig. 2(a). The trapping potential is harmonic in the axial direction $z$, whereas in the transverse directions it has approximately a quartic form close to the center, and maxima separated by $\sim 2.5 \mu \mathrm{m}$. From the measured three-dimensional intensity distribution [Fig. 2(a)], we obtain minimum trap barriers (saddle point along the escape cones) of around $0.6 \mathrm{mK}$ [Fig. 2(b)]. When the variation of the field is substantial over a length scale comparable to the atom size, the trapping potential $U_{n l j m_{j}}(\boldsymbol{R})$ depends strongly on the specific Rydberg state. This is illustrated in the cuts along the transverse direction for different Rydberg states displayed in Fig. 2(c). When the orbital radius $\sim n^{2} a_{0}$ of the atom becomes comparable to the trap size (here, for $n \sim 120$ ), the potential does not have a local minimum any longer, and Rydberg atoms are then repelled from the BOB trap.

The experimental sequence is shown in Fig. 3. First, ${ }^{87} \mathrm{Rb}$ atoms from a magneto-optical trap are loaded in the optical tweezers (with temperatures in the range

(a)

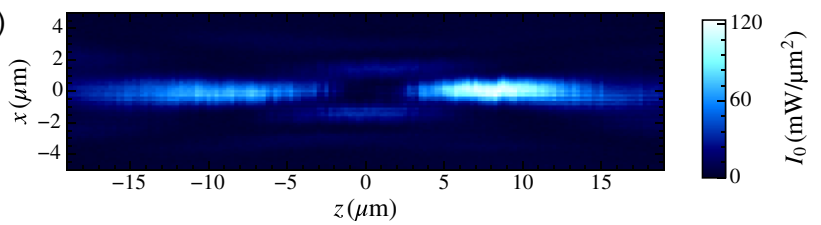

(b)
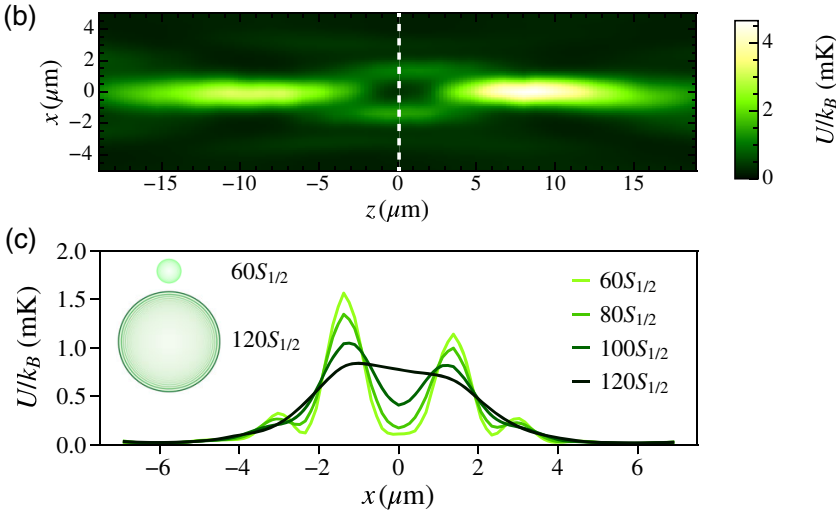

FIG. 2. (a) Example of measured light intensity distribution near the focal plane. (b) Trapping potential for the $84 S_{1 / 2}$ Rydberg state calculated from Eq. (1) and the intensity distribution shown in (a). (c) Cuts of the trapping potential along $z=0$ [dashed line in (b)] for different $n S_{1 / 2}$ Rydberg states. Schematics of the $60 S$ and $120 S$ Rydberg orbitals (to scale) are shown as insets. 
3-130 $\mu \mathrm{K}$ depending on the experiment). We detect the occupancy of each trap by collecting the fluorescence of the atoms at $780 \mathrm{~nm}$ with an electron-multiplying CCD camera in $20 \mathrm{~ms}$. After successful detection of a filled trap, atoms are optically pumped in the ground state $|g\rangle=\mid 5 S_{1 / 2}$, $\left.F=2, m_{F}=2\right\rangle$, the tweezers are switched off, and a twophoton STImulated Raman Adiabatic Passage (STIRAP) prepares the target Rydberg state $|r\rangle$ with $\sim 90 \%$ efficiency. We then switch on the BOB trap for a variable duration $\tau$. Finally, a Rydberg deexcitation pulse, resonant with the $|r\rangle-5 P_{1 / 2}$ transition, brings the population in $|r\rangle$ back to the ground state $|g\rangle$ via spontaneous decay from $5 P_{1 / 2}$, and the atom is imaged by fluorescence. The result of one of these experiments is plotted in Fig. 3. Without the BOB trap (red curve), this release and recapture experiment can be used to measure single-atom temperatures [43]. The recapture probability for atoms in $84 S_{1 / 2}$ is reduced by a factor of 10 after $30 \mu$ s. From this curve we extract a temperature of $\sim 130 \mu \mathrm{K}$. With the BOB trap (blue curve), the recapture probability is drastically enhanced: only $30 \%$ of the atoms are lost in the same amount of time, consistent with the expected Rydberg lifetime (see below). We do not observe any heating of the atoms after they are transferred back to the ground-state tweezers.

Following a procedure similar to the one in Ref. [44], we determined the typical radial oscillation frequency of the Rydberg atom in the trap. After the atoms are loaded into

(a)

(b)
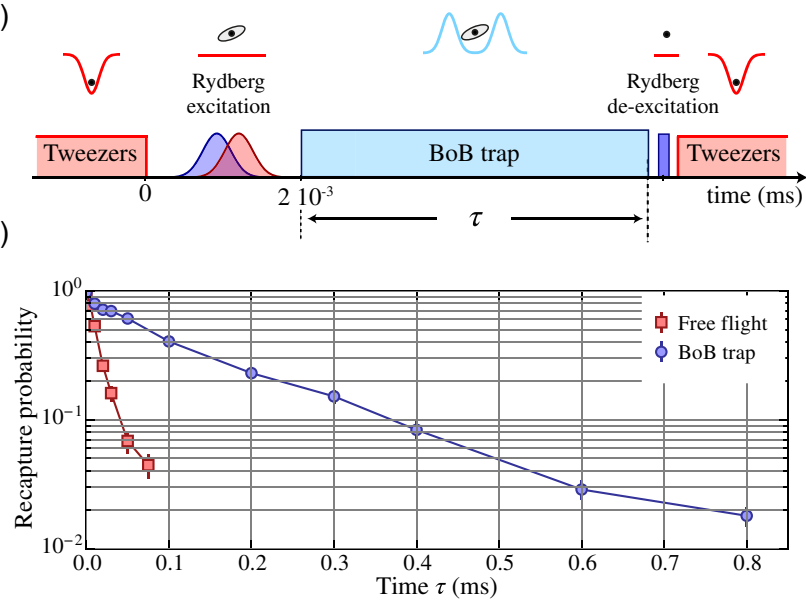

FIG. 3. (a) Time sequence of the experiment. Ground-state atoms are initially loaded in optical tweezers. The tweezers are then switched off for Rydberg excitation. After $2 \mu$ s the BOB trap is applied for a variable duration $\tau$. Following Rydberg deexcitation in free flight, the tweezers are switched on again and the atoms are imaged. As the BOB trap is strongly repulsive for ground-state atoms, only atoms that were in the Rydberg state before deexcitation are recaptured. (b) Recapture probability for atoms excited to the $84 S_{1 / 2}$ state as a function of the trapping time $\tau$ (disks). The recapture curve in the absence of a BOB trap (squares) is shown for comparison. Error bars are the standard error of the mean. the BOB trap, we excite breathing oscillations by switching off the trap for three microseconds. During this time the atom leaves the bottom of the potential. We then switch on the trap again for a variable time $T$, during which the atom oscillates in the trap with a typical frequency $\omega_{\mathrm{BOB}}$. The recapture probability after a fixed switch-off time oscillates with a frequency $2 \omega_{\text {BOB }}$. For atoms excited to the $84 S_{1 / 2}$ state, we observe oscillations in the recapture probability that are well reproduced by Monte Carlo simulations taking into account the experimental parameters, where the atom trajectories inside the trap are computed classically [45]. For a trap depth of $\sim 0.6 \mathrm{mK}$ the atoms oscillate with a measured frequency of $\omega_{\mathrm{BOB}} /(2 \pi)=59 \pm 3 \mathrm{kHz}$, in excellent agreement with the simulations, which predict $59 \mathrm{kHz}$ for the same parameters. The necessary power for efficient trapping depends on the trap size, the initial temperature of the atoms, and the target Rydberg state. For our smallest trap (with maxima separated by $\sim 2 \mu \mathrm{m}$ in the radial direction), and temperatures of $\sim 3 \mu \mathrm{K}$, we need only $20 \mathrm{~mW}$ of power to reach our higher trapping efficiencies for the $60 S_{1 / 2}$ Rydberg state, which shows that it will be possible to scale this technique up to large arrays of BOB traps.

To evaluate the quality of the $\mathrm{BOB}$ potential as a trap for Rydberg atoms, we measured the trap-decay times for different Rydberg states. The recapture probability as a function of the time spent in the BOB trap is shown for the $84 S_{1 / 2}$ state in Fig. 4(a). An exponential fit to the data (dashed line) gives a $1 / e$ decay time of $222 \pm 3 \mu \mathrm{s}$, in excellent agreement with the calculated lifetime $228 \mu$ s of this state at $300 \mathrm{~K}$ [46-48]. The solid line is the result of a Monte-Carlo simulation of the atomic trajectories in the BOB trap potential [calculated using Eq. (1) and the measured light intensity distribution] and taking into account not only radiative decay, but also the possibility for some energetic atoms to spill over the trap barrier. All the parameters entering the simulation were given their experimentally measured values [45]. Figure 4(b) shows (a)

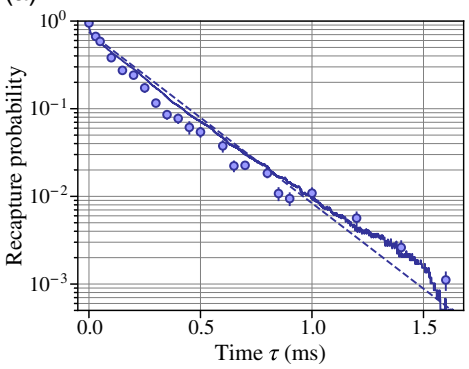

(b)

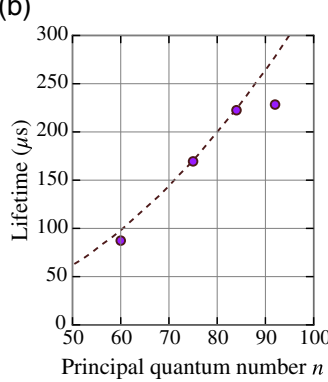

FIG. 4. (a) Recapture probability of a $84 S_{1 / 2}$ Rydberg atom, as a function of the time spent in the BOB trap, showing a roughly exponential decay with lifetime $222 \pm 3 \mu$ s (dashed line). The solid line is the result of a simulation without any adjustable parameter (see text). (b) Measured lifetimes of various $n S_{1 / 2}$ states; the dashed line is the theoretical value [46]. 
the results of the same experiment for other $n S_{1 / 2}$ states; the measured decay times match almost perfectly the expected lifetime (dashed line) up to $n \sim 90$. This indicates that atom losses are mainly due to Rydberg decay, and that other processes, such as photoionization [22,35,49], are negligible. We also performed the same measurement for the $84 P_{1 / 2}$ and $82 D_{3 / 2}$ states (not shown) and obtained lifetimes $\sim 20 \%$ shorter than their theoretical values.

Beyond $n=90$, the extension of the radial wave function becomes comparable to the trap size and trapping is less efficient. As a consequence, some atoms can escape the trap, and the loss rate is significantly higher than expected from the Rydberg lifetime alone. Beyond $n \sim 100$ the potential does not have a local minimum and the Rydberg atoms are quickly expelled from the BOB traps. Monte Carlo simulations [45] show that for $n=60$, atoms can be lost only through the narrow escape cones of the BOB trap, while for $n=100$, we observe much more pronounced losses over the radial barrier due to the small depth of the trap.

We now investigate the performance of our BOB trap for quantum simulation tasks by studying its compatibility with one- and two-qubit operations. For low principal quantum numbers $(n<60)$, the trapping potential corresponds approximately to the one of a free electron, and it is therefore almost independent of $n$ or the atom's angular momenta. For higher Rydberg states the wave function of the electron must be considered in the convolution (1). Most quantum simulation experiments, however, involve only couplings to adjacent Rydberg levels, for which changes in the squared radial part of the wave function play only a minor role in the effective potential. The angular part, in turn, is identical for states with different quantum number $l$, but same $j, m_{j}$ (e.g., for $\mid n S_{1 / 2}$, $\left.m_{j}=1 / 2\right\rangle$ and $\left.\left|n P_{1 / 2}, m_{j}=1 / 2\right\rangle\right)$. This "quasi-magic" trapping condition strongly suppresses differential light shifts in Rabi oscillations between different trapped Rydberg states. In a first experiment, we analyze the coherence in the spin manipulation of a single trapped atom. We apply the BOB trap for a total time of $50 \mu \mathrm{s}$. We let the atoms move in the trapping potential during $\sim 35 \mu$ s and then we drive microwave Rabi oscillations between the states $|\uparrow\rangle=\left|82 D_{3 / 2}, m_{j}=3 / 2\right\rangle$ and $|\downarrow\rangle=$ $\left|83 P_{1 / 2}, m_{j}=1 / 2\right\rangle$, while the atoms are still confined [Fig. 5(a)]. We observe spin flip oscillations without appreciable damping, despite the two states having different angular wave functions. The constant finite contrast of the oscillations is mainly due to the excitation efficiency and the limited lifetime of the involved Rydberg states. We measured similar Rabi flopping curves even for atom temperatures as high as $130 \mu \mathrm{K}$, where the atoms explore a large volume of the BOB trap.

As a final illustration of the usefulness of our trapping scheme for quantum simulation, we measure spinexchange dynamics driven by dipole-dipole interaction (a)
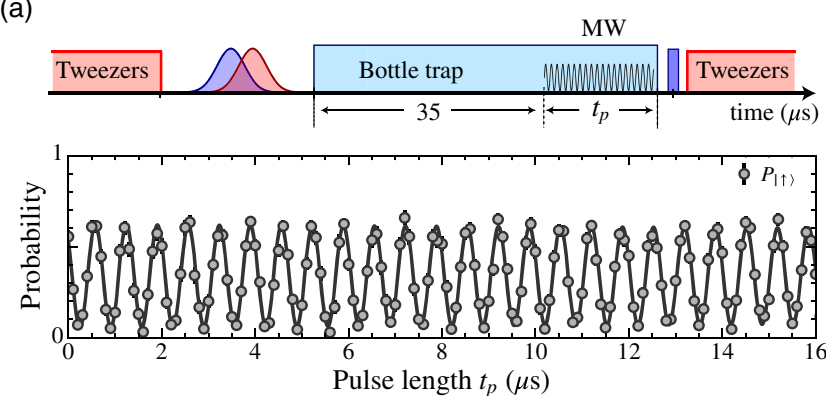

(b)

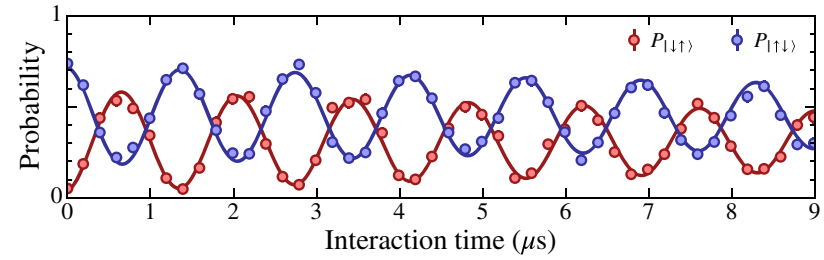

FIG. 5. (a) Rabi oscillation between the states $|\uparrow\rangle=\mid 82 D_{3 / 2}$, $\left.m_{j}=3 / 2\right\rangle$ and $|\downarrow\rangle=\left|83 P_{1 / 2}, m_{j}=1 / 2\right\rangle$. The total trapping time is $50 \mu \mathrm{s}$. (b) Excitation-hopping oscillations in the population of the pair states $|\uparrow \downarrow\rangle$ and $|\downarrow \uparrow\rangle$, mediated by the dipoledipole interaction between the Rydberg states $|\uparrow\rangle$ and $|\downarrow\rangle$ at a distance of $40 \mu \mathrm{m}$. The temperature of the atoms is $\sim 3 \mu \mathrm{K}$. Solid lines are damped sine fits to the data. Error bars represent the standard error of the mean and are most often smaller than the symbol size.

between two atoms. We create two traps separated by a distance of $R=40 \mu \mathrm{m}$ [Fig. 5(b)]. Immediately after loading the Rydberg atoms in the BOB traps, we use a resonant microwave field and local addressing [50] to prepare the first atom in $|\uparrow\rangle$ and the second one in $|\downarrow\rangle$. In these two Rydberg states, the atoms are resonantly coupled by a dipole-dipole interaction of strength $U_{\mathrm{dd}} / h=C_{3} / R^{3} \sim 0.4 \mathrm{MHz}$, and the dynamics is governed by an $X Y$-spin Hamiltonian $H=U_{\mathrm{dd}}\left(\sigma_{1}^{+} \sigma_{2}^{-}+\sigma_{1}^{-} \sigma_{2}^{+}\right)$, where $\sigma_{i}^{ \pm}$are the Pauli matrices for atom $i=1,2$. We observe coherent spin exchange between the pair states $|\uparrow \downarrow\rangle$ and $|\downarrow \uparrow\rangle$. This experiment demonstrates the compatibility of our Rydberg trapping scheme with quantum simulations using current experimental setups, with levels of contrast and damping comparable or better that previously achieved $[23,50]$.

In conclusion, we have demonstrated laser trapping of individual Rydberg atoms in microscopic potentials and shown the suitability of such traps for quantum information tasks. This trapping scheme can be extended to larger arrays with moderate laser power for atoms which can be cooled to the motional ground state before Rydberg excitation [51,52]. In addition, the ponderomotive traps can, in principle, be used for circular Rydberg atoms in a cryogenic environment, opening the door to unprecedented trapping times [53]. The results presented here are suited for experiments that occur entirely in the Rydberg manifold. For quantum logic gates or for quantum simulation 
involving also the ground state in addition to Rydberg states, it would be possible, by using shorter wavelength trapping light, to realize BOB traps that satisfy the groundRydberg "magic" condition and would minimize heating and decoherence rates [35,54].

This work benefited from financial support by the EU (FET-Flag 817482, PASQUANS), by the Région Île-deFrance in the framework of DIM SIRTEQ (project CARAQUES), and by the IXCORE-Fondation pour la Recherche. T. B. acknowledges the support of the European Marie Skłodowska-Curie Actions (H2020-MSCA-IF-2015 Grant No. 701034).

Note added.-Two-dimensional trapping of circular Rydberg atoms in the ponderomotive potential of a hollow laser beam has been observed recently at Collège de France [55].

*Present address: Institute for Molecular Science, National Institutes of Natural Sciences, Myodaiji, Okazaki 4448585, Japan.

[1] D. Jaksch, J. I. Cirac, P. Zoller, S. L. Rolston, R. Côté, and M. D. Lukin, Phys. Rev. Lett. 85, 2208 (2000).

[2] M. Saffman, T. G. Walker, and K. Mølmer, Rev. Mod. Phys. 82, 2313 (2010).

[3] H. Weimer, M. Muller, I. Lesanovsky, P. Zoller, and H. P. Büchler, Nat. Phys. 6, 382 (2010).

[4] M. Saffman, J. Phys. B 49, 202001 (2016).

[5] J. D. Pritchard, D. Maxwell, A. Gauguet, K. J. Weatherill, M. P. A. Jones, and C. S. Adams, Phys. Rev. Lett. 105, 193603 (2010).

[6] Y. O. Dudin and A. Kuzmich, Science 336, 887 (2012).

[7] O. Firstenberg, T. Peyronel, Q.-Y. Liang, A. V. Gorshkov, M. D. Lukin, and V. Vuletić, Nature (London) 502, 71 (2013).

[8] D. Tiarks, S. Baur, K. Schneider, S. Dürr, and G. Rempe, Phys. Rev. Lett. 113, 053602 (2014).

[9] H. Gorniaczyk, C. Tresp, J. Schmidt, H. Fedder, and S. Hofferberth, Phys. Rev. Lett. 113, 053601 (2014).

[10] T. Wilk, A. Gaëtan, C. Evellin, J. Wolters, Y. Miroshnychenko, P. Grangier, and A. Browaeys, Phys. Rev. Lett. 104, 010502 (2010).

[11] L. Isenhower, E. Urban, X. L. Zhang, A. T. Gill, T. Henage, T. A. Johnson, T. G. Walker, and M. Saffman, Phys. Rev. Lett. 104, 010503 (2010).

[12] Y.-Y. Jau, A. M. Hankin, T. Keating, I. H. Deutsch, and G. W. Biedermann, Nat. Phys. 12, 71 (2016).

[13] H. Levine, A. Keesling, A. Omran, H. Bernien, S. Schwartz, A. S. Zibrov, M. Endres, M. Greiner, V. Vuletić, and M. D. Lukin, Phys. Rev. Lett. 121, 123603 (2018).

[14] C. J. Picken, R. Legaie, K. McDonnell, and J. D. Pritchard, Quantum Sci. Technol. 4, 015011 (2019).

[15] P. Schauß, M. Cheneau, M. Endres, T. Fukuhara, S. Hild, A. Omran, T. Pohl, C. Gross, S. Kuhr, and I. Bloch, Nature (London) 491, 87 (2012).
[16] P. Schauß, J. Zeiher, T. Fukuhara, S. Hild, M. Cheneau, T. Macrì, T. Pohl, I. Bloch, and C. Gross, Science 347, 1455 (2015).

[17] J. Zeiher, J. Y. Choi, A. Rubio-Abadal, T. Pohl, R. van Bijnen, I. Bloch, and C. Gross, Phys. Rev. X 7, 041063 (2017).

[18] H. Labuhn, D. Barredo, S. Ravets, S. de Léséleuc, T. Macrì, T. Lahaye, and A. Browaeys, Nature (London) 534, 667 (2016).

[19] H. Bernien, S. Schwartz, A. Keesling, H. Levine, A. Omran, H. Pichler, S. Choi, A. S. Zibrov, M. Endres, M. Greiner, V. Vuletić, and M. D. Lukin, Nature (London) 551, 579 (2017).

[20] H. Kim, Y. J. Park, K. Kim, H.-S. Sim, and J. Ahn, Phys. Rev. Lett. 120, 180502 (2018).

[21] S. de Léséleuc, V. Lienhard, P. Scholl, D. Barredo, S. Weber, N. Lang, H. P. Büchler, T. Lahaye, and A. Browaeys, Science 365, 775 (2019).

[22] M. Saffman and T. G. Walker, Phys. Rev. A 72, 022347 (2005).

[23] D. Barredo, H. Labuhn, S. Ravets, T. Lahaye, A. Browaeys, and C. S. Adams, Phys. Rev. Lett. 114, 113002 (2015).

[24] S. de Léséleuc, D. Barredo, V. Lienhard, A. Browaeys, and T. Lahaye, Phys. Rev. A 97, 053803 (2018).

[25] A. Ramos, K. Moore, and G. Raithel, Phys. Rev. A 96, 032513 (2017).

[26] U. D. Jentschura, P. J. Mohr, J. N. Tan, and B. J. Wundt, Phys. Rev. Lett. 100, 160404 (2008).

[27] D. B. Cassidy, Eur. Phys. J. D 72, 53 (2018).

[28] J.-H. Choi, J. R. Guest, A. P. Povilus, E. Hansis, and G. Raithel, Phys. Rev. Lett. 95, 243001 (2005).

[29] D. A. Anderson, A. Schwarzkopf, R. E. Sapiro, and G. Raithel, Phys. Rev. A 88, 031401(R) (2013).

[30] A. D. Bounds, N. C. Jackson, R. K. Hanley, R. Faoro, E. M. Bridge, P. Huillery, and M. P. A. Jones, Phys. Rev. Lett. 120, 183401 (2018).

[31] E. Vliegen, S. D. Hogan, H. Schmutz, and F. Merkt, Phys. Rev. A 76, 023405 (2007).

[32] S. D. Hogan and F. Merkt, Phys. Rev. Lett. 100, 043001 (2008).

[33] S. E. Anderson, K. C. Younge, and G. Raithel, Phys. Rev. Lett. 107, 263001 (2011).

[34] L. Li, Y. O. Dudin, and A. Kuzmich, Nature (London) 498, 466 (2013).

[35] S. Zhang, F. Robicheaux, and M. Saffman, Phys. Rev. A 84, 043408 (2011).

[36] S. Zhang, Ph. D. thesis, University of Wisconsin-Madison, 2012.

[37] R. Ozeri, L. Khaykovich, and N. Davidson, Phys. Rev. A 59, R1750 (1999).

[38] P. Xu, X. He, J. Wang, and M. Zhan, Opt. Lett. 35, 2164 (2010)

[39] D. Barredo, V. Lienhard, S. de Léséleuc, T. Lahaye, and A. Browaeys, Nature (London) 561, 79 (2018).

[40] J. L. Chaloupka, Y. Fisher, T. J. Kessler, and D. D. Meyerhofer, Opt. Lett. 22, 1021 (1997).

[41] The residual light intensity at the origin is below $1 \%$ of the maximal intensity of the beam.

[42] S. K. Dutta, J. R. Guest, D. Feldbaum, A. Walz-Flannigan, and G. Raithel, Phys. Rev. Lett. 85, 5551 (2000).

[43] C. Tuchendler, A. M. Lance, A. Browaeys, Y. R. P. Sortais, and P. Grangier, Phys. Rev. A 78, 033425 (2008). 
[44] Y. R. P. Sortais, H. Marion, C. Tuchendler, A. M. Lance, M. Lamare, P. Fournet, C. Armellin, R. Mercier, G. Messin, A. Browaeys, and P. Grangier, Phys. Rev. A 75, 013406 (2007).

[45] See Supplemental Material at http://link.aps.org/ supplemental/10.1103/PhysRevLett.124.023201 for more details.

[46] I. I. Beterov, I. I. Ryabtsev, D. B. Tretyakov, and V. M. Entin, Phys. Rev. A 79, 052504 (2009).

[47] N. Šibalić, J. Pritchard, C. Adams, and K. Weatherill, Comput. Phys. Commun. 220, 319 (2017).

[48] M. Archimi, C. Simonelli, L. Di Virgilio, A. Greco, M. Ceccanti, E. Arimondo, D. Ciampini, I. Ryabtsev, I. Beterov, and O. Morsch, Phys. Rev. A 100, 030501 (2019).

[49] R. M. Potvliege and C. S. Adams, New J. Phys. 8, 163 (2006).
[50] S. de Léséleuc, D. Barredo, V. Lienhard, A. Browaeys, and T. Lahaye, Phys. Rev. Lett. 119, 053202 (2017).

[51] A. M. Kaufman, B. J. Lester, and C. A. Regal, Phys. Rev. X 2, 041014 (2012).

[52] J. D. Thompson, T. G. Tiecke, A. S. Zibrov, V. Vuletić, and M. D. Lukin, Phys. Rev. Lett. 110, 133001 (2013).

[53] T. L. Nguyen, J. M. Raimond, C. Sayrin, R. Cortiñas, T. Cantat-Moltrecht, F. Assemat, I. Dotsenko, S. Gleyzes, S. Haroche, G. Roux, T. Jolicoeur, and M. Brune, Phys. Rev. X 8, 011032 (2018).

[54] M. J. Piotrowicz, M. Lichtman, K. Maller, G. Li, S. Zhang, L. Isenhower, and M. Saffman, Phys. Rev. A 88, 013420 (2013).

[55] R. G. Cortiñas, M. Favier, B. Ravon, P. Méhaignerie, Y. Machu, J. M. Raimond, C. Sayrin, and M. Brune, arXiv:1911.02316. 